Above $570 \cdot \mathrm{K}$ the $a$ axes (Fig. 3), the mean-square displacement $u(\perp c)$ of $\mathrm{Li}(2)$ (Fig. $2 b)$ and the occupation probability of $\mathrm{Li}(2)$ (Fig. 4) all deviate from a linear temperature dependence. These deviations are also probably caused by the anharmonic thermal motion of the $\mathrm{Li}(2)$ ions.

The number of $\mathrm{Li}(2)$ ions vibrating with large amplitudes in the direction of neighboring occupied $\mathrm{Li}(2)$ sites has increased such that now the $\mathrm{Li}(2)-\mathrm{Li}(2)$ interaction results in an additional expansion of $a$.

The usual structure factor includes only harmonic thermal vibrations. Therefore, it cannot describe the strong anharmonic thermal vibrations and the corresponding electron density distribution of the $\mathrm{Li}(2)$ sites correctly. Likewise, corresponding least-squares programs adapt the usual structure parameters to an assumed harmonic distribution. This results in an overproportional decrease of the occupation probability and an overproportional increase of $u(\perp c)$ of $\mathrm{Li}(2)$, whereas the defect concentration at the $\mathrm{Li}(2)$ positions has probably not changed, but only the number of highly excited $\mathrm{Li}(2)$ ions has increased.

The relations between anharmonic thermal vibrations and ionic conductivity described here have also been observed in AgI in a similar way (Cava, Reidinger \& Wuensch, 1977).

We acknowledge many fruitful discussions with Professor A. Rabenau and Dr W. Weppner, MaxPlanck-Institut für Festkörperforschung, Stuttgart, and with Professor R. A. Huggins, currently at the Max-
Planck-Institut, Stuttgart, on sabbatical leave from Stanford University. We thank R. A. Huggins and W. Weppner for the careful and critical reading of the manuscript.

\section{References}

Alpen, U. von, Bell, M. F., Wichelhaus, W., Cheung, K, Y. \& Dudley, G. J. (1978). Electrochim. Acta. In the press.

Alfen, U. von, Rabenau, A. \& Talat, G. H. (1977). Appl. Phys. Lett. 30, 621-623.

Boukamp, B.A. \& Huggins, R. A. (1976). Phys. Lett. A, 58, $231-233$.

Cava, R. J., Reidinger, F. \& Wuensch, B. J. (1977). Solid State Commun. 24, 411-416.

Hong, H. Y. P. (1978). Mater. Res. Bull. 13, 117-124.

International Tables for X-ray Crystallography (1974). Vol. IV. Birmingham: Kynoch Press

Larson, A. C. (1967). Acta Cryst. 23, 664-665.

Rabenau, A. \& Schulz, H. (1976). J. Less-Common Met. 50, 155-159.

Schönherr, E., Müller, G. \& Winkler, E. (1978). J. Cryst. Growth, 43, 469-472.

Schulz, H. \& Schwarz, K. H. (1978). Acta Cryst. A34, 999-1005.

Schwarz, K. H. \& Schulz, H. (1978). Acta Cryst. A34, 994-999.

Wahl, J. \& Holland, U. (1978). Solid State Commun. 27, 237-241.

ZinTl, E. \& Brauer, G. (1935). Z. Elektrochem. 41, 102107.

Acta Cryst. (1979). A35, 314-316

\title{
On the Choice of Elastic Constants in Evaluating Thermal Diffuse Scattering Corrections
}

\author{
BY G. DE WITH*
}

Chemical Physics Laboratory, Twente University of Technology, P.O. Box 217, Enschede, The Netherlands

(Received 5 June 1978; accepted 28 September 1978)

\begin{abstract}
The elastic stiffness constants for the calculation of the thermal diffuse scattering correction of X-ray reflexions should be the isothermal ones. However, adiabatic ones are mostly used. It is shown that, despite a relatively large difference between both types of elastic stiffness constants for pyrazine, the resulting thermal diffuse scattering correction is hardly affected.
\end{abstract}

\footnotetext{
* Present address: Philips Research Laboratories, Eindhoven, The Netherlands.
}

At present thermal diffuse scattering (TDS) corrections of X-ray reflexions are usually evaluated in the socalled long-wave approximation. In this approximation the TDS correction factor $\alpha$ is given by (Cochran, 1969; Harada \& Sakata, 1974)

$$
\alpha=(2 \pi)^{-3} \int_{\text {scan }} J(\mathbf{q}) \mathrm{d} \mathbf{q},
$$

with

$$
J(\mathbf{q})=\frac{k_{B} T}{q^{2}} \sum_{i, l} s_{i}\left(\mathbf{A}^{-1}\right)_{j k} s_{l}
$$

(C) 1979 International Union of Crystallography 
and

$$
A_{i j}=\sum_{k, l} C_{k i l j} q_{k} q_{l}
$$

In this formula $q_{i}$ denote the wavevector components, $k_{B}$ the Boltzmann constant, $T$ the temperature, $s_{i}$ the scattering vector components and $C_{i j k l}$ the elastic stiffness constants. The corrected net intensity is then given by

$$
I_{\text {corr. }}=I /\left(1+\alpha^{\prime}\right),
$$

where $\alpha^{\prime}$ is that part of $\alpha$ which has not been taken into account by the background correction procedure.

The expression for $\alpha$ can be derived from harmonic as well as from pseudo-harmonic lattice dynamics. In the harmonic case there is no ambiguity for the choice of the elastic stiffness constants $C_{i j k l}$. Because of the lack of thermal expansion of a crystal in this approximation the adiabatic $\left(C_{i j k l}^{A}\right)$ and isothermal $\left(C_{i j k l}^{T}\right)$ values of the elastic stiffness constants are identical. This, however, is not true in the pseudo-harmonic case. It has been shown by Hahn $(1961,1962)$ that the longwave behaviour in the pseudo-harmonic approximation is determined by the $C_{i j k l}^{T}$. If, as usual, the elastic stiffness constants are determined by ultrasonic techniques adiabatic values are obtained. The difference between the $C_{i j k l}^{T}$ and $C_{i j k l}^{A}$ can be calculated with aid of the elements of the inverse of the matrix $C_{i j k l}$. Denoting these elements - the elastic compliance constants - by $S_{i j k l}$, the following relation holds

$$
S_{i j k l}^{A}-S_{i j k l}^{T}=-\beta_{i j} \beta_{k l} T V_{\text {spec }} / c_{p},
$$

where $V_{\text {spec }}$ is the specific volume, $c_{p}$ the isobaric heat capacity and $\beta_{i j}$ an element of the thermal expansion tensor. The resulting difference is small for most inorganic compounds. For molecular crystals this is not true. For naphthalene at $300 \mathrm{~K}$ an average difference $R_{c}=\sum\left|C_{i j k l}^{A}-C_{i j k l}^{T}\right| / \sum\left|C_{i j k l}^{T}\right|=11.2 \%$ can be calculated from the data given by Kitaigorodsky (1973).

Table 1. Elastic stiffness constants for pyrazine in $\mathrm{Mb}$ $\left(10^{12}\right.$ dyne $\left./ \mathrm{cm}^{2}\right)$ at room temperature

$\begin{array}{lcccc}i j^{(a)} & C^{T^{(b)}} & C^{A^{(c)}} & C^{A}-C^{T} & \% \\ 11 & 0.2270 & 0.2300 & 0.0030 & 1 \cdot 3 \\ 22 & 0.0510 & 0.0538 & 0.0028 & 5.5 \\ 33 & 0.0930 & 0.1060 & 0.0130 & 14.0 \\ 44 & 0.0260 & 0.0260 & - & - \\ 55 & 0.0150 & 0.0150 & - & - \\ 66 & 0.0290 & 0.0290 & - & - \\ 12 & 0.0140 & 0.0169 & 0.0029 & 20.7 \\ 13 & 0.0550 & 0.0612 & 0.0062 & 11.3 \\ 23 & 0.0245 & 0.0305 & 0.0060 & 24.5\end{array}$

(a) Here indexing $i j$ is according to the matrix notation of the tensor $C_{i j k l}$ (see for example Nye, 1969).

(b) Data taken from Reynolds (1973).

(c) $C_{44}^{A}, C_{55}^{A}$ and $C_{66}^{A}$ are identical to the corresponding $C^{T}$ values as a consequence of the orthorhombic symmetry.
An accurate determination of the charge distribution in pyrazine (de With, Harkema \& Feil, 1976) has been made. The influence of the TDS correction on the determination of the charge distribution was discussed. In order to check the influence of the relatively large difference between the $C_{i j k l}^{A}$ and $C_{i j k l}^{T}, \alpha^{\prime}$ was evaluated for several reflexions of pyrazine for both sets of $C_{i j k l}$. The $C_{i j k l}^{A}$ were calculated from the $C_{i j k l}$ as determined by Reynolds (1973) at room temperature using inelastic neutron scattering (assuming that his experiment yielded the $C_{i j k l}^{T}$ ones). Both sets are given in Table 1, together with the absolute and relative differences. For $V_{\text {spec }}$ we used $63.27 \times 10^{3} \mathrm{~mm}^{3} \mathrm{~mol}^{-1}$ as calculated from the molecular weight and density of the crystal, obtained from the unit-cell volume. The volume of $c_{p}$ was measured by differential scanning calorimetry (DSC) and amounts to $5.79 \pm 0.05 \mathrm{~J} \mathrm{~K}^{-1}$ $\mathrm{mol}^{-1}$ at room temperature. The expansion tensor for pyrazine is given by de With (1976). The resulting $R_{c}$ is $6 \cdot 3 \%$.

For 24 reflexions, distributed uniformly through reciprocal space $\alpha^{\prime}$ was calculated with both sets of elastic stiffness constants. In this calculation conditions like crystal orientation, detector aperture and scan width were chosen in accordance with the experimental ones of pyrazine (de With, Harkema \& Feil, 1976).

Least-squares fitting the $\alpha^{\prime}$ values obtained from the $C_{i j k l}^{A}$ against those as calculated from the $C_{i j k l}^{T}$ yields a straight line through the origin with a slope of $0.975 \pm$ 0.002 . The correlation between both sets of $\alpha^{\prime}$ values is $\mathbf{9 9 . 9 9 \%}$ (standard error 0.003 ). This means that taking $C_{i j k l}^{A}$ values instead of $C_{i j k l}^{T}$ values yields $\alpha^{\prime}$ values which are $2.5 \%$ too small, a negligible error in view of all approximations.

Only recently it has been established that, although absolute values of $\alpha^{\prime}$ are not quite correct as compared with $\alpha^{\prime}$ values calculated from lattice dynamics without further approximation, the trend and order of magnitude are certainly correct (Kroon, 1977).

Many thanks are due to $\mathrm{Mr}$ G. Oversluizer (Utrecht) and Dr A. Schuyff (Utrecht) for measuring the $c_{p}$.

This research has been carried out under the auspices of the Foundation for Fundamental Research on Matter by Electron and X-rays (FOMRE) and with aid from the Netherlands Organization for Advancement of Pure Research (ZWO).

\section{References}

Cochran, W. (1969). Acta Cryst. A25, 95-101.

HAHN, H. (1961). Z. Phys. 165, 569-600.

HAHN, H. (1962). Inelastic Scattering of Neutrons in Solids and Liquids. IAEA, Vienna, Vol. 1, pp. 37-48. 
Harada, J. \& Sakata, M. (1974). Acta Cryst. A30, 77-82.

KITAIGORODSKY, A. I. (1973). Molecular Crystals and Molecules. New York: Academic Press.

Kroon, P. A. (1977). Thesis, State Univ. of Groningen, The Netherlands.

NYE, J. F. (1969). Physical Properties of Crystals. Oxford: Clarendon Press.
Reynolds, P. A. (1973). J. Chem. Phys. 59, 2777-2786.

WITH, G. DE (1976). J. Appl. Cryst. 9, 502.

With, G. DE, HaRkema, S. \& Feil, D. (1976). Acta Cryst. B32, 3178-3184.

Acta Cryst. (1979). A35, 316-318

\title{
X-ray Attenuation Coefficients of Graphite in the Range 0.40 to $1.54 \AA$
}

\author{
By A. A. Berry ANd J. L. LAwrence \\ School of Physical Sciences, University of St Andrews, St Andrews, Fife, Scotland
}

(Received 3 May 1978; accepted 30 September 1978)

\begin{abstract}
The mass attenuation coefficients of graphite have been measured at 23 different wavelengths in the range 0.40 to $1.54 \AA$. Above $1 \AA$, the coefficient is proportional to $\lambda^{2 \cdot 88}$.
\end{abstract}

\section{Introduction}

A method of measuring accurate X-ray attenuation coefficients has been described by Lawrence \& Mathieson (1976) and has been used to measure the attenuation coefficient of silicon for Mo $K \alpha$ and $\mathrm{Cu} K \alpha$ radiations (Lawrence, 1977). The method consists of measuring the intensity, $I$, of a monochromatic beam of $\mathrm{X}$-rays transmitted through a regular specimen of crystal of thickness $t$ as the angle between the incident beam and the normal to the crystal face, $\varphi$, is varied. Thus, if $I_{0}$ is the incident intensity,

$$
I=I_{0} \exp (-\mu t / \cos \varphi)
$$

and a plot of $\ln I$ versus $1 / \cos \varphi$ will yield values of $\mu t$ from which $\mu$ can be deduced. $\mu$ is of course dependent on the wavelength of the radiation and the presence of any spectral components in the main beam will be detected by departures from linearity of the plot.

In this study, the method was applied to an investigation of the wavelength dependence of the absorption coefficients of graphite in the wavelength range 0.40 to $1 \cdot 54 \AA$.

\section{Experimental}

The crystal used was a single crystal of pyrolytic graphite of uniform thickness and rectangular crosssection $20 \times 10 \mathrm{~mm}$. Its average thickness over the surface traversed by the X-ray beam was measured

0567-7394/79/020316-03\$01.00 using a linear differential transducer, carefully calibrated using slip gauges, and found to be $t=0.922$ (3) $\mathrm{mm}$.

The apparatus used was similar to that employed in the measurement of the attenuation coefficients of silicon (Lawrence, 1977), the main beam being first diffracted by the (111) planes of a near-perfect silicon crystal and collimated by two circular apertures, of radius $0.5 \mathrm{~mm}$, separated by $150 \mathrm{~mm}$, one aperture being $60 \mathrm{~mm}$ away from the X-ray source. The crystal was centred on a spectrometer which was capable of being rotated through a minimum of 3 " of arc.

The wavelengths used were narrow ranges of the white radiation from a tungsten target, certain tungsten characteristic $L$ lines, the $K \alpha$ and $K \beta$ lines of copper and silver targets and the $K \alpha$ line of a molybdenum target. In each case the wavelength was measured by determining the $2 \theta$ angle through which the graphite crystal was rotated in moving from the 0002 to the 0002 reflecting position, assuming $c$ (graphite) $=$ $6.7079 \AA$ (Nelson \& Riley, 1945). The diffraction profiles of these reflections were fairly wide, about $10^{\prime}$ of arc, and were found to be independent of wavelength. On the instrument used, the centres of each peak could be determined to $\pm 12^{\prime \prime}$, giving an uncertainty in each characteristic wavelength of $0.001 \AA$. [The wavelengths quoted for the characteristic lines will not necessarily correspond exactly to the accepted values since, for the copper, molybdenum and silver targets, different proportions of the $\alpha_{1}$ and $\alpha_{2}$ lines have been gathered by the collimator and, for the tungsten characteristic lines, more than one line could be present.] For the wavelengths obtained from the white radiation of the tungsten tube, the divergence through the collimator was 0.0014 rad, giving a wavelength spread of less than $0.01 \AA$.

The $\varphi$ angles were chosen such that $1 / \cos \varphi=n$, where $n$ took all integer and half-integer values between (C) 1979 International Union of Crystallography 